\title{
Fuzzy Predictive Control of a Solar Power Plant
}

\author{
Andrés Flores, Doris Sáez, Member, IEEE, Juan Araya, Manuel Berenguel, and Aldo Cipriano, Senior Member, IEEE
}

\begin{abstract}
This paper presents the application of fuzzy predictive control to a solar power plant. The proposed predictive controller uses fuzzy characterization of goals and constraints, based on the fuzzy optimization framework for multi-objective satisfaction problems. This approach enhances model based predictive control (MBPC) allowing the specification of more complex requirements. A brief description of the solar power plant and its simulator is given. Basic concepts of predictive control and fuzzy predictive control are introduced. Two fuzzy predictive controllers using different membership functions are designed for a solar power plant, and they are compared with a classical predictive controller. The simulation results show that the fuzzy MBPC formulation, based on a well proven successful algorithm, gives a greater flexibility to characterize the goals and constraints than classical control.
\end{abstract}

Index Terms-Fuzzy constraints, fuzzy goals, fuzzy optimization, predictive control, solar power plant.

\section{INTRODUCTION}

$\mathbf{O}$ NE OF THE main characteristics of a solar power plant is that the primary energy source (solar radiation) cannot be manipulated. Besides, the solar radiation intensity depends on daily and seasonal cycle variations, like clouds, atmospheric humidity, and air transparency. This justifies the relevance of solar power plant control.

This study considers the Acurex distributed collector solar field, located at the Almería Solar Platform, Almería, Spain. In this solar power plant, the main control goal is to maintain constant outlet oil temperature, despite the operation conditions changes, by manipulating the field oil flow. To maintain constant outlet temperatures during the day while the solar conditions changes, significant flow variations are required. This produces considerable variations in the dynamics of the process. Hence, conventional control algorithms based on a simplified model of the process, for example the linear quadratic Gaussian (LQG) regulator, proves to be ineffective [1]. However LQG/linear transfer recovery (LTR) regulator gives better results even when the working conditions are far from the ones that LQG controller has been designed for [2].

Due to its nonlinear characteristics, the Acurex solar collector field has been used as an experimental platform for the application of many modern control algorithms [3].

Manuscript received February 5, 2002; revised April 15, 2004. This work was supported by FONDECYT Chile under Grant 1990101.

A. Flores, J. Araya, and A. Cipriano are with the Departamento de Ingeniería Eléctrica, Pontificia Universidad Católica de Chile, Casilla 306, Santiago 22, Chile (e-mail: aciprian@ing.puc.cl).

D. Sáez was with the Departamento de Ingeniería Eléctrica, Pontificia Universidad Católica de Chile, Casilla 306, Santiago 22, Chile. She is now with the Departamento de Ingeniería Eléctrica, Universidad de Chile, Casilla 412-3, Santiago, Chile (e-mail: dsaez@ing.uchile.cl).

M. Berenguel is with the Departamento de Lenguajes y Computación, Universidad de Almería, E-04120 Almería, Spain (e-mail: beren@ual.es).

Digital Object Identifier 10.1109/TFUZZ.2004.839658
In 1992, Camacho et al. [4] described a self-tuning proportional-integral (PI) controller for this solar plant, based on a pole assignment approach. In order to compensate the measured disturbances, a feedforward controller is included. The self-tuning controller is capable to deal with changes in the operating conditions of the plant.

Later, several model based predictive control algorithms have been implemented and experimentally tested. For example, Camacho et al. [5] presented an adaptive generalized predictive controller (GPC). The algorithm is based on reaction curve modeling that uses recursive least squares estimation. The proposed controller is successfully compared with a self-tuning PI controller [4].

Camacho et al. [6] proposed a gain scheduling GPC, based on the fact that the controller's parameters depend on the same variables that define the operating condition. In this case, the dynamics of the field are mainly conditioned to the oil flow, which can be used to change the controller's parameters. The main advantage of this controller compared to the previous adaptive algorithms is that the controller parameters are fixed.

Camacho and Berenguel [7] described a GPC algorithm based on a nonlinear model. In this case, the nonlinear model is used to generate an estimation of the free response of the process, due to past control actions and disturbances. This term is combined with the forced response, which is calculated using a linear model. The nonlinear model allows the controller to deal with changes in the dynamics of the process. Camacho et al. [8] described an extension of the mentioned algorithm, in which the free response is based on a neural network obtaining a control scheme that shows very good performance.

In 1995, Rubio et al. [9] presented a fuzzy logic controller for the solar plant. The fuzzy controller is based on rules obtained using expert knowledge of the process. Subsequently, Gordillo et al. [10] proposed a genetic design of a fuzzy logic controller. The genetic algorithm is used to optimize the parameters of the fuzzy controller.

Cardoso et al. [11] and Henriques et al. [12] described a fuzzy switching supervisor PID control strategy for the solar plant. The fuzzy supervisor controller measures actual data available from the plant providing a way to switch between several fixed controllers. Additionally, the local PID controllers are offline tuned, with a dynamic recurrent neural network with pole placement. In 1999, Henriques et al. [13] proposed the same idea but the fuzzy switching is made using $c$-means clustering.

Distinctly, Juuso et al. [14] presented a fuzzy PI controller applied to the solar plant. The results show that the fuzzy algorithm is very robust in various difficult operating conditions.

Pickardt [15], [16] described an indirect adaptive controller LQG and GPC for the solar plant. The algorithm uses three or five linear auto regressive with moving average and exogenous inputs (ARMAX) models and contains an online identification procedure to determine and to update the corresponding model 


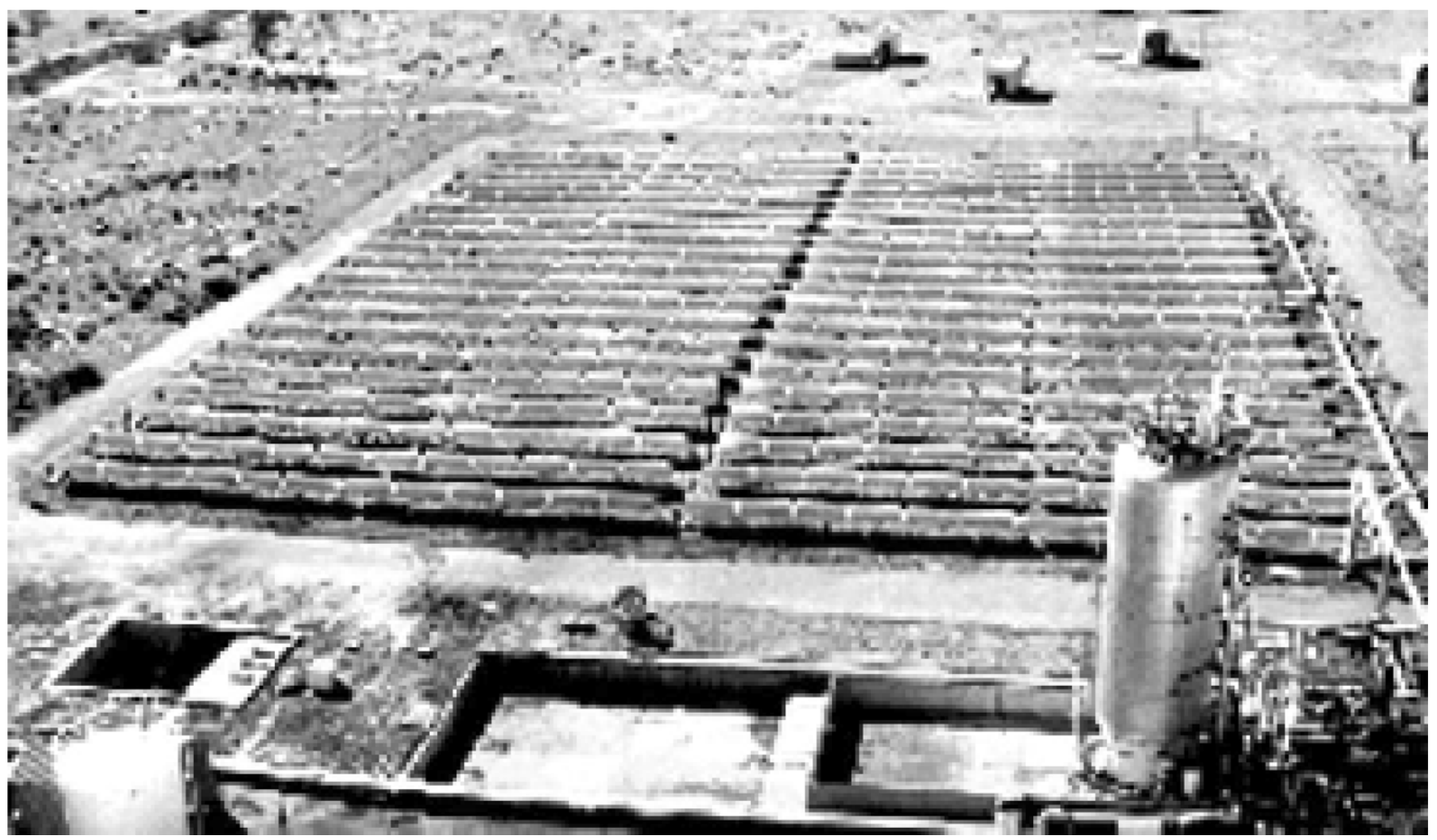

Fig. 1. Panoramic view of the Acurex solar collectors.

of the operating point. In this case, adaptive LQG and GPC are designed and compared, obtaining similar satisfactory results.

Johansen et al. [17] proposed a gain-scheduled control for the solar plant. In this case, the algorithm uses high-order local linear auto regressive with exogenous inputs (ARX) models and the local linear controllers are designed based on pole placement. This author's forecoming work describes a distributed model based controller for the solar plant. Stability of the closed-loop is proven using Lyapunov conditions.

In order to compare fuzzy predictive control with classical predictive control, this paper considers a simple MBPC controller based on a linear model of the solar collector field. The system is kept around the operation point and no "hard" constraints are imposed to the process. A fuzzy predictive controller is designed using the same linear model, but applying fuzzy characterization to goals for the controlled variable error and constraints over the manipulated variable.

This paper starts with a brief description of the solar power plant, including the process and simulator description. Next, a classical predictive control algorithm for the solar power plant is described. The fuzzy goals and constraints characterization of the predictive algorithm is explained. Finally, the application to the solar power plant simulator is shown.

\section{SOLAR POWER Plant}

\section{A. Process Description}

The considered solar power plant is located in Almería, Spain. The main objective of the solar plant, based on a distributed collector field, is to collect solar energy by heating oil passing through the field (see Fig. 1).

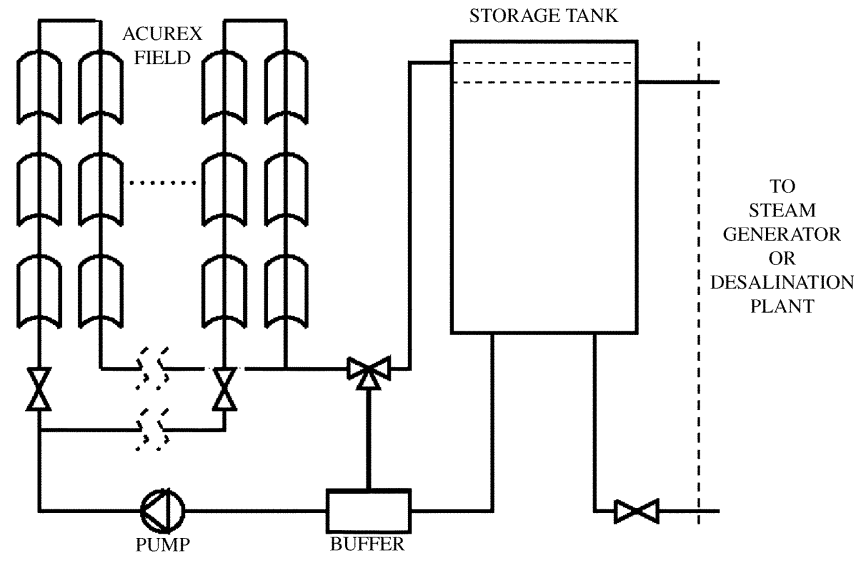

Fig. 2. Schematic diagram of the Acurex distributed collector field.

As shown in Fig. 2, the field consists of 480 collectors. These collectors are arranged in 20 rows that form ten parallel loops and lie along an east-west axis [4].

The field is also provided with a tracking system, which causes the mirrors to revolve around an axis parallel to the pipe, to enable the varying inclination of the sun to be followed. The cold inlet oil is extracted from the bottom of a storage tank and is passed through the field using a pump located in the field inlet. This fluid is heated and then introduced back into the storage tank to be used for electricity generation.

\section{B. Simulator Description}

The plant can be described by a set of nonlinear distributed parameter equations obtained from energy and mass balance. 
The dynamic of the process is given by the following main partial differential equations [18]:

$$
\begin{aligned}
\rho_{m} c_{m} A_{m} \frac{\partial T_{m}}{\partial t}= & I \eta_{0} D-h_{L} G\left(T_{m}-T_{a}\right) \\
& -L h_{T}\left(T_{m}-T_{f}\right) \\
\rho_{f} c_{f} A_{f} \frac{\partial T_{f}}{\partial t}+\rho_{f} c_{f} V \frac{\partial T_{f}}{\partial x}= & L h_{T}\left(T_{m}-T_{f}\right)
\end{aligned}
$$

where $m$ and $f$ subscripts are related to the metal and oil fluid $(1 / \mathrm{s})$, respectively. Also

$t$ time (s);

$x \quad$ position (m);

$\rho \quad$ oil density $\left(\mathrm{kg} / \mathrm{m}^{3}\right)$;

$c \quad$ specific heat of oil $(\mathrm{J} / \mathrm{kgK})$;

A cross-section of the pipeline $\left(\mathrm{m}^{2}\right)$;

$T$ outlet oil temperature $\left({ }^{\circ} \mathrm{C}\right)$;

I solar radiation $\left(W / m^{2}\right)$;

$\eta_{o} \quad$ optical efficiency;

$D \quad$ width of the mirror (m);

$h_{L} \quad$ overall thermal loss coefficient $\left(W / m^{2} K\right)$;

$G \quad$ exterior diameter of the pipeline (m);

$T_{a} \quad$ environment temperature $\left({ }^{\circ} \mathrm{C}\right)$;

$L \quad$ inner diameter of the pipeline (m),

$h_{T}$ metal-fluid transmission coefficient $\left(W / m^{2} K\right)$,

$V \quad$ volumetric oil flow rate $\left(\mathrm{m}^{3} / \mathrm{s}\right)$.

In order to develop the solar power plant simulator, a hundred distributed parameter models representing different sections of the collector field are merged into a model of the plant [3].

\section{Classical Predictive Control Algorithms}

Model based predictive control (MBPC) involves a complete family of controllers whose basic concepts are (see Fig. 3).

1) Use of an explicit model to predict the process outputs at discrete future time instants, over a prediction horizon.

2) Computation of a sequence of future control actions through the optimization of a certain objective function, which considers given operation constraints and desired reference trajectories for processes' outputs.

3) Receding horizon strategy, i.e., the optimization process is repeated at each sampling instant and the first action in the calculated control sequence is applied [19].

These three characteristics allow MBPC to handle multivariable, nonminimum phase, open loop unstable and nonlinear processes, with a long time delay or including constraints for manipulated and/or controlled variables, if necessary.

At instant $t$, future process outputs are predicted using an explicit model over the prediction horizon $N_{p}$. The predictions depend on known values of the manipulated and controlled variables and the future control actions $u(t+j \mid t)$ over the control horizon $N_{u}$ (where the notation $u(t+j \mid t)$ indicates that the future control signals depend on the conditions at time $t$ ). It is assumed that $N_{u} \leq N_{p}$ and that $u(t+j \mid t)$ remains constant for $j=N_{u}, \ldots, N_{p}$. The constant $N_{p}$ is related to the response time of the process and $N_{u}$ is usually chosen to be equal to the model order.

The control sequence is obtained from the optimization of an objective function, which describes the goals that the control

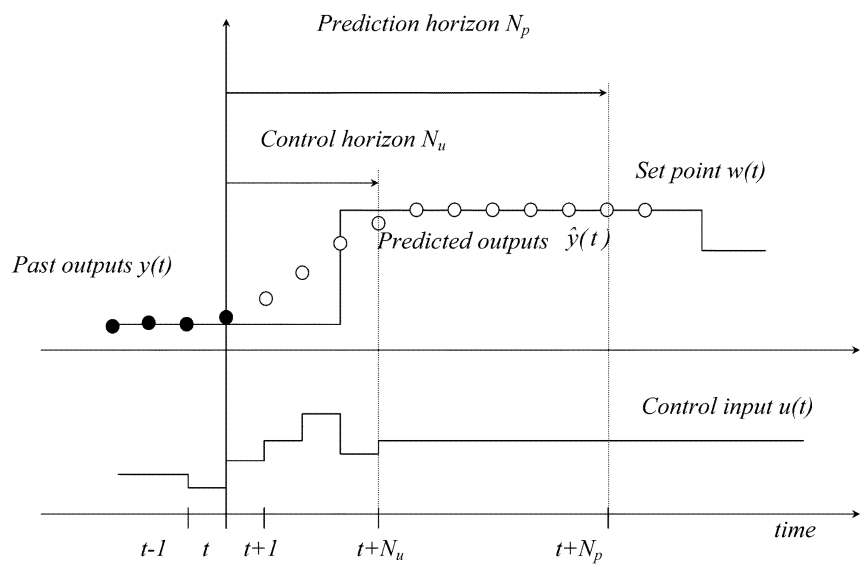

Fig. 3. MBPC strategy.

strategy wants to achieve. The optimization process can include "hard" or "soft" constraints if they are considered in the objective function [20]. Classical MBPC uses an objective function that minimizes the control effort $\Delta u$ and the error between the predicted outputs and the set points $w$, during the prediction and the control horizon, respectively, as is shown in (3) [19]

$$
\begin{aligned}
J=\sum_{j=N_{1}}^{N_{p}} \delta(j)(\hat{y}(t+j \mid t) & -w(t+j))^{2} \\
& +\sum_{j=1}^{N_{u}} \lambda(j)(\Delta u(t+j-1 \mid t))^{2}
\end{aligned}
$$

where $\hat{y}(t+j \mid t)$ is the expected value of the predicted output at instant $t+j$ with known history up to time $t$. Parameters $\delta(j)$ and $\lambda(j)$ weight every term involved in the optimization problem. These values can change while the time is evolving to priorize, for example, the final error or the transient period. The value of the parameter $N_{1}$ usually is settled as the "dead time" of the control action over the process, but if it is necessary, it can be greater.

For linear unconstrained systems, this tractable convex optimization problem can be solved analytically. However, in general applications it is common to consider constraints or nonlinearities of the process, and in these cases the problem must be solved by using numerical (usually iterative) methods. Further considerations about this subject will be discussed in Section IV.

\section{FuZZy GOALS AND CONSTRAINTS CHARACTERIZATION}

Model-based fuzzy predictive control combines the basic idea of traditional predictive control using an objective function which results from the aggregation of different fuzzy criteria based on operator knowledge about the process and its requirements. This principle is represented in Fig. 4 [21].

As in classical MBPC, predictions for future values of process outputs for a given control sequence are obtained through linear or nonlinear models and a receding horizon strategy is applied. Because in reality the cost function is generally only an approximation of the desired control performance, this new methodology suggests the use of fuzzy goals and constraints instead of the typical quadratic criterion. 


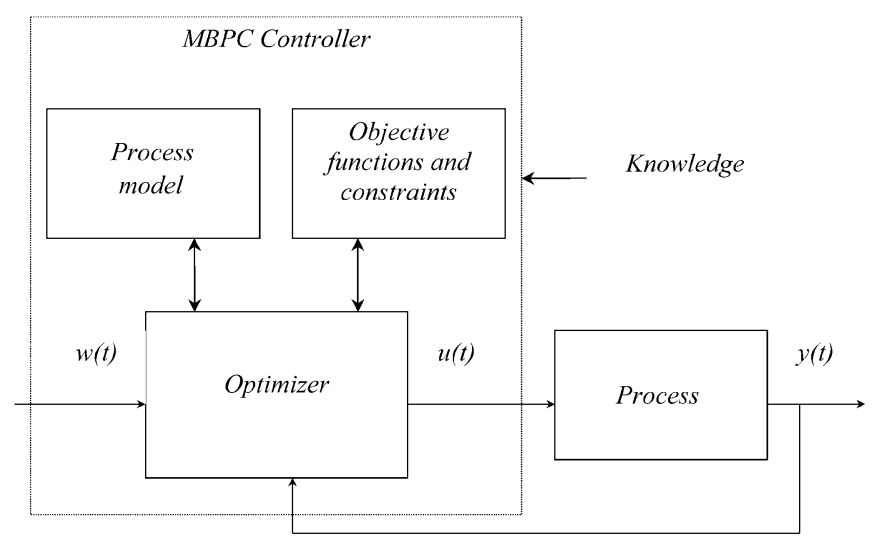

Fig. 4. Classical and fuzzy MBPC basic schemes. (a)

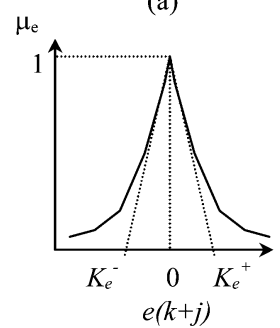

(b)

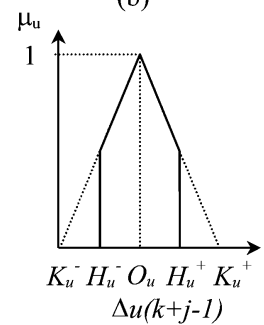

Fig. 5. Membership functions representing satisfaction. (a) Fuzzy goal consisting on minimizing an error. (b) Fuzzy constraint that keeps the change in the control action in a desired range.

There is more flexibility in the definition of the optimization criteria than in the case of traditional predictive control; in fact, both the fuzzy membership function for the goals or constraints and their aggregation operator can be arbitrary selected.

It is even possible to add some desirable objectives that play also an important role in the process, but they do not have the same relevance compared to the strict goals and constraints of classical MBPC [22].

The goals and constraints that define a fuzzy predictive control problem can be represented by a fuzzy membership function as is shown in Fig. 5. This problem can be solved using the fuzzy decision framework introduced by Bellman and Zadeh [23]. For each instant of time, there exists $n$ fuzzy goals $G_{1}, G_{2}, \ldots, G_{n}$ and $m$ fuzzy constraints $C_{1}, C_{2}, \ldots, C_{m}$. Then, it is possible to define a global objective by intersecting every fuzzy membership function over the time horizons defined in the fuzzy MBPC problem [see (4)]

$$
\mu_{J}=\left(G_{1} \cap \cdots \cap G_{n}\right) \cap\left(C_{1} \cap \cdots \cap C_{m}\right) .
$$

It is important to notice that the fuzzy decision framework approach treats goals and constraints similarly, therefore the relative weighting of goals and constraints is restricted by the choice of the fuzzy membership functions.

The optimization must be made for the whole future control sequence and in that case the optimization problem becomes

$$
\operatorname{Max}_{\Delta u} \mu_{J}=\operatorname{Max}_{\Delta u}\left(G_{1} \cap \cdots \cap G_{n}\right) \cap\left(C_{1} \cap \cdots \cap C_{m}\right) .
$$

The maximization of $\mu_{J}$ defines a fuzzy decision problem, which consists on achieving the greatest degree in which fuzzy criteria, fuzzy goals, and fuzzy constraints are satisfied simultaneously. For example, if the fuzzy intersection is evaluated using the min $t$-norm the problem is to maximize the minimum degree of satisfaction among fuzzy goals and fuzzy constraints. The "hardness" of the intersection can be changed choosing a different $t$-norm.

In order to define the minimization problem, a fuzzy complement is applied to the cost function of (5), resulting in the following equivalent problem:

$$
\operatorname{Min}_{\Delta u} \overline{\mu_{J}}=\operatorname{Min}_{\Delta u}\left(\overline{G_{1}} \cup \cdots \cup \overline{G_{n}}\right) \cup\left(\overline{C_{1}} \cup \cdots \cup \overline{C_{m}}\right) .
$$

Different operators like the algebraic product, maximum, sum, or other $t$-norm can be used to make the fuzzy aggregation. The election of the method must consider the desired softness in the control outputs, shape of utilized membership functions and available computational resources. Literature suggests using the Yager operator [24]. This operator is defined by the following expressions:

$$
\begin{aligned}
t_{\text {norm }}(a, b) & =\max \left\{0,1-\left((1-a)^{p}+(1-b)^{p}\right)^{1 / p}\right\} \\
t_{\text {co-norm }}(a, b) & =\min \left\{1,\left(a^{p}+b^{p}\right)^{1 / p}\right\} .
\end{aligned}
$$

The parameter $p, p \in(0, \infty)$, adjusts the degree of the fuzzy aggregation. A greater $p$ represents a "harder" fuzzy aggregation.

In order to compare the minimization problem of (6) with the classical MBPC formulation, membership functions like those of Fig. 5 and the Yager $t$-norm will be considered [see (7)]. With these considerations, an equivalent problem to the one in (3) can be solved in fuzzy predictive control by minimizing the following objective function:

$$
\begin{aligned}
\mu_{J}^{\prime}=\sum_{j=N_{1}}^{N_{p}}\left(\bar{\mu}_{e}(\hat{y}(t+j \mid t)\right. & -w(t+j)))^{p} \\
& +\sum_{j=1}^{N_{u}}\left(\bar{\mu}_{u}(\Delta u(t+j-1 \mid t))\right)^{p}
\end{aligned}
$$

and $\mu_{J}=\min \left\{1, \mu_{J}^{\prime} 1 / p\right\}$.

Because of nonlinearity in the process model and/or the decision function, the optimization problem is usually nonconvex and cannot be solved using standard optimization algorithms such as quadratic programming (QP). In order to solve the optimization problem represented in (9) using a gradient method like sequential quadratic programming (SQP), the Yager operator must be relaxed, not limiting its value to a fuzzy number between 0 and 1 ; the relaxation consists in modifying expression (7) to consider only the second argument of the maximum operator. 


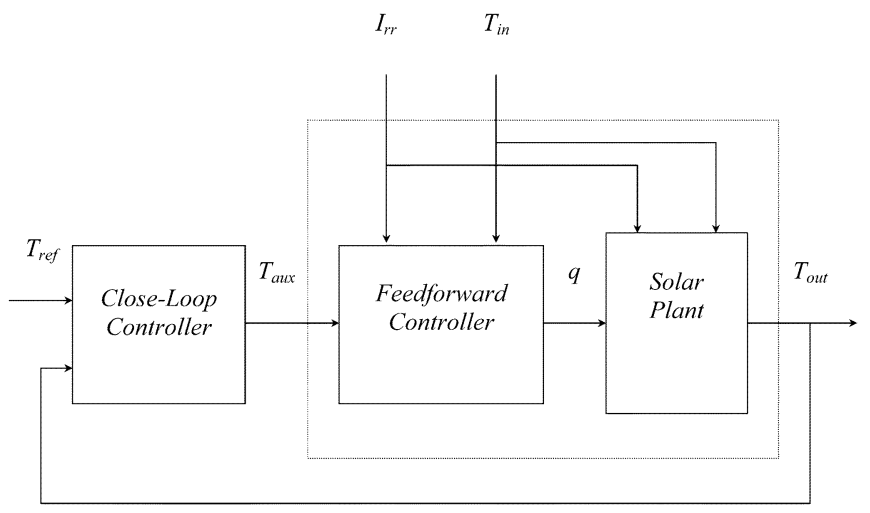

Fig. 6. Feedback-feedforward control system.

TABLE I

MAIN SYSTEM VARIABLES

\begin{tabular}{ccc}
\hline Symbol & Variable & Unit \\
\hline$T_{\text {ref }}$ & Reference outlet oil temperature & ${ }^{\circ} \mathrm{C}$ \\
$T_{\text {aux }}$ & Feedforward input temperature & ${ }^{\circ} \mathrm{C}$ \\
$q$ & Oil flow (manipulated variable) & $l / s$ \\
$T_{\text {out }}$ & Outlet oil temperature (controlled variable) & ${ }^{\circ} \mathrm{C}$ \\
$T_{\text {in }}$ & Inlet oil temperature & ${ }^{\circ} \mathrm{C}$ \\
$I_{r r}$ & Solar radiation & $\mathrm{W} / \mathrm{m}^{2}$ \\
\hline
\end{tabular}

\section{ApPlication to the Solar Power Plant}

\section{A. Problem Statement}

The main control goal in the solar plant is to keep the outlet oil temperature as close as possible to a given reference temperature. This should be achieved through changes in the field oil flow, despite the disturbances that affect the system. These disturbances are mainly the solar radiation, the inlet oil temperature and the environment temperature.

In order to apply and compare the controllers described in Sections III and IV to the solar plant, this paper considers a simple predictive controller that uses a linear model around an operation point [3]. This control scheme includes a feedforward controller, shown in Fig. 6, to compensate the effect of measured disturbances, solar radiation, and inlet oil temperature. In addition, the main variables are listed in Table I.

The output of the feedforward controller is calculated from the following expression [3], [18]:

$$
q=\frac{0.7869 I_{\mathrm{rr}}-0.485\left(T_{\mathrm{aux}}-151.5\right)-80.7}{T_{\mathrm{aux}}-T_{\mathrm{in}}} .
$$

The simulation study presented in this paper considers two specific disturbance profiles, which are shown in Fig. 7 [3]. The solar radiation profile varies according to the year season, daytime, and geometry of the distributed collector field. Additionally both disturbances are filtered using a moving-average filter that considers the eight past samples.

\section{B. Classical Predictive Controller}

MBPC can be implemented considering a linear model for the combined dynamic system, plant and the feedforward controller (see Fig. 6), for an operation oil flow of 6 1/s. This combined system leads to the following controlled auto regressive with integrated moving average (CARIMA) model [3]:

$$
A\left(z^{-1}\right) T_{\text {out }}(t)=B\left(z^{-1}\right) T_{\text {aux }}(t-1)+\frac{e(t)}{\Delta}
$$

where $\Delta=1-z^{-1}$ and

$$
\begin{aligned}
A\left(z^{-1}\right)= & 1-1.5681 z^{-1}+0.5934 z^{-2} \\
B\left(z^{-1}\right)= & 0.0612+0.0018 z^{-1}-0.0171 z^{-2}+0.0046 z^{-3} \\
& +0.0005 z^{-4}+0.0101 z^{-5}-0.0064 z^{-6} \\
& -0.015 z^{-7}-0.0156 z^{-8}
\end{aligned}
$$

The sampling period of the model and controller is $39 \mathrm{~s}$. Considering the cost function of (3) the horizon parameters are set to $N_{1}=1, N_{p}=15$ and $N_{u}=15$. The weights used are $\delta(j)=1$ and $\lambda(j)=7$, for $j=1$ to 15 . Although the oil flow is limited to the range $2 \mathrm{l} / \mathrm{s}$ to $12 \mathrm{l} / \mathrm{s}$, the study is performed around the operating condition of $6 \mathrm{l} / \mathrm{s}$ of oil flow.

In this case, the MBPC algorithm produces the $T_{\text {aux }}$ sequence that minimizes the cost function, without considering active constraints. The first value of this sequence is applied to the feedforward controller that calculates an oil flow that takes in account the measured disturbances.

As Fig. 8(a) shows, the classical MBPC follows the outlet oil temperature set-point despite the disturbances profiles (see Fig. 7). It is clear that the system response present some oscillations. Two possible reasons for this behavior are the presence of disturbances and the incomplete dynamic modeling due to a simple linear model obtained through excitation of the plant. For the given disturbance profiles, it is more likely the oscillations are the outcome to the lack of complete dynamic modeling. In fact, this plant exhibits antiresonance behavior [3] as a consequence of resonance modes that are excited when fast responses are required. The characteristic frequencies of these resonance modes are different depending on the operating point. The linear model used for control purposes in this paper takes into account these phenomena when operating near $61 / \mathrm{s}$. As it is shown in Fig. 8, oscillations are lesser as the oil flow approaches this value, and higher far from this nominal value.

\section{Fuzzy Predictive Controller}

The structure of the fuzzy predictive controller is similar to the MBPC presented in Section V, this means the model and horizons are the same. The cost function is given by (9). The Yager $t$-norm with $p=2$ is used as fuzzy aggregation operator, and the minimization problem is solved using SQP.

As explained in Section IV, the fuzzy characterization of goals and constraints is achieved with fuzzy membership functions, with $\mu_{e}(\cdot)$ for the error $E T_{\text {out }}=T_{\text {out }}-T_{\text {ref }}$ and $\mu_{u}(\cdot)$ for the $T_{\text {aux }}$ variation. These functions are defined over the set of possible values for both variables. This is a natural straightforward way to go from classical predictive control to a fuzzy one. 
(a)

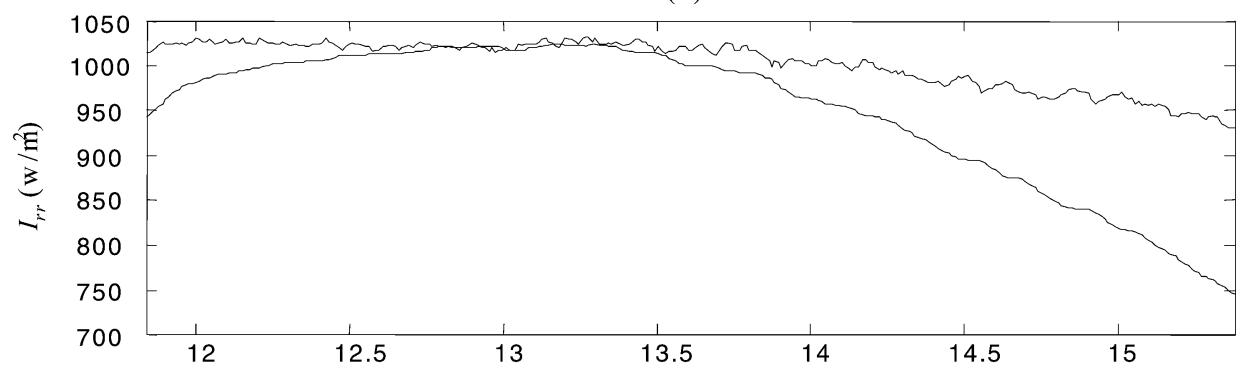

(b)

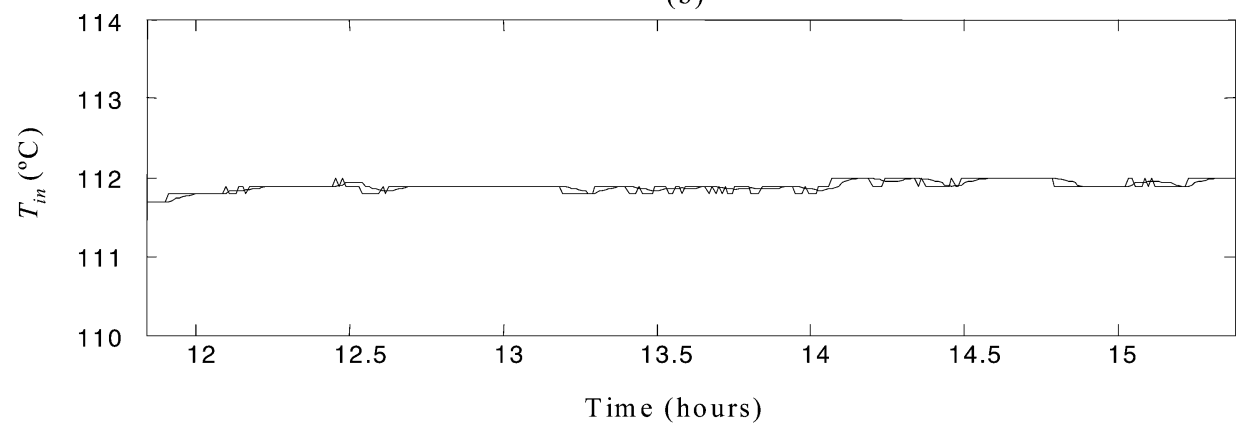

Fig. 7. Disturbance profiles. (a) Solar radiation. (b) Oil inlet temperature.

(a)

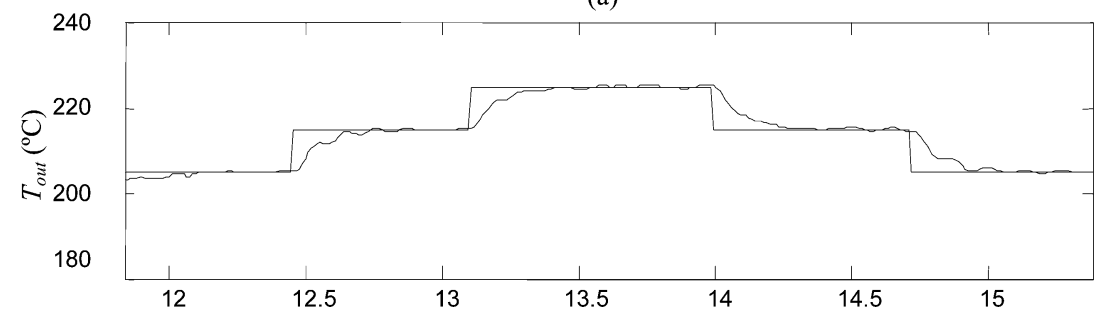

(b)

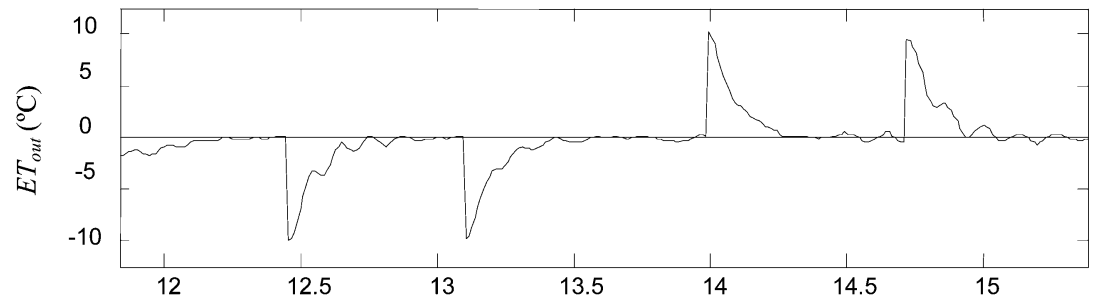

(c)

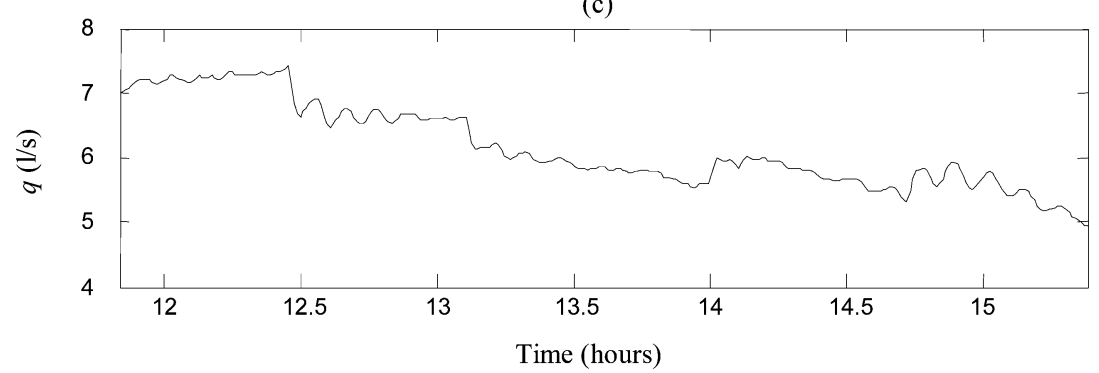

Fig. 8. Simulation results for classical MBPC. (a) Outlet oil temperature. (b) Error in the outlet oil temperature. (c) Oil flow.

A general triangular-shaped membership function is defined by three parameters indicating the inflection points of the function. The following definition will be used:
The following membership functions will be considered (Fig. 9):

$$
\operatorname{trimf}(x, a, b, c)= \begin{cases}\frac{x-a}{b-a}, & \text { if } a \leq x<b \\ \frac{x-c}{b-c}, & \text { if } b \leq x \leq c \\ 0, & \text { otherwise. }\end{cases}
$$

$$
\begin{aligned}
\mu_{e}\left(E T_{\text {out }}\right) & =\operatorname{trimf}\left(E T_{\text {out }},-25,0,25\right) \\
\mu_{u}\left(T_{\text {aux }}\right) & =\operatorname{trimf}\left(T_{\text {aux }},-9.5,0,9.5\right) .
\end{aligned}
$$



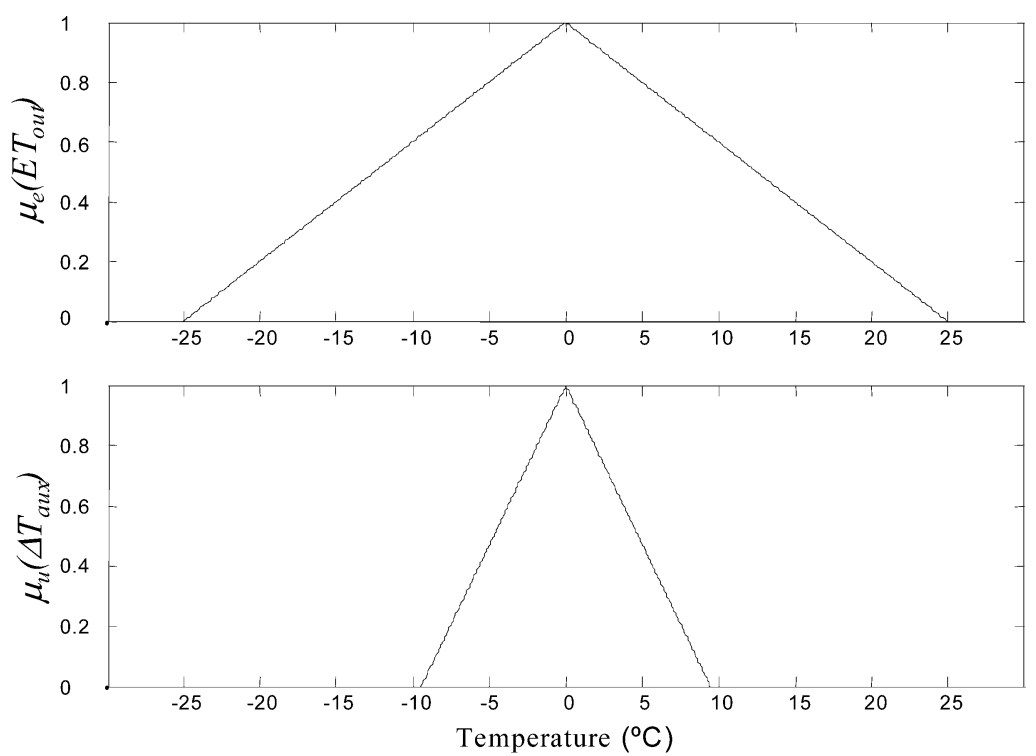

Fig. 9. Triangular-shaped membership functions for $T_{\text {out }}$ error and $T_{\text {aux }}$ variation.

(a)

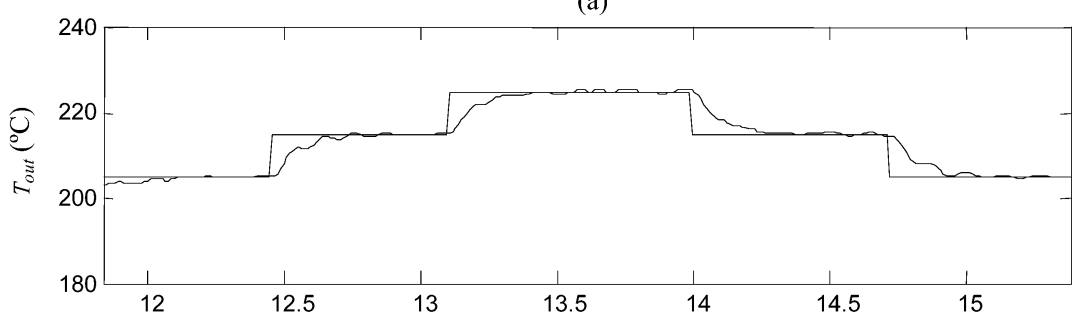

(b)

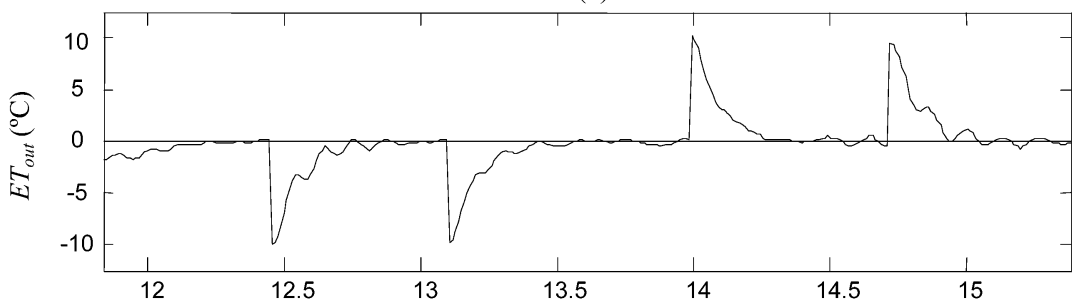

(c)

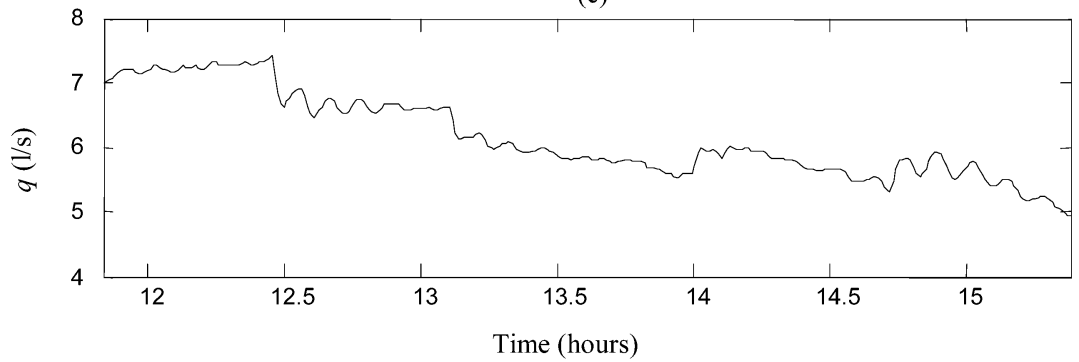

Fig. 10. Simulation results for fuzzy MBPC with triangular-shaped membership functions. (a) Outlet oil temperature. (b) Error in the outlet oil temperature. (c) Oil flow.

The parameters of these membership functions were chosen to replicate the behavior of the classical predictive control with the weights mentioned in Section V and for the considered operation ranges.

The simulation results for fuzzy predictive control with triangular-shaped membership functions are shown in Fig. 10. Comparing Figs. 8 and 10, the responses of the system with either one of the controllers seem to be identical. The explanation to this is the fact that the slopes of the fuzzy membership functions were fixed according to the weights of classical MBPC.

The similarities of both controllers can be further noticed in the histograms shown in Fig. 11, which presents the $T_{\text {out }}$ error distribution for both cases.

As it has been mentioned before, model-based fuzzy predictive control offers several degrees of flexibility and one of them is the election of membership functions. In the next paragraphs, 
(a)

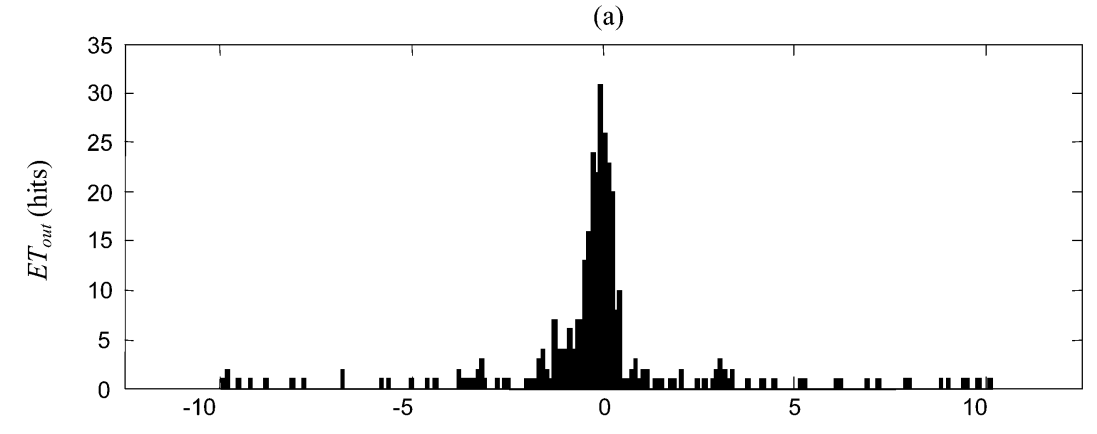

(b)

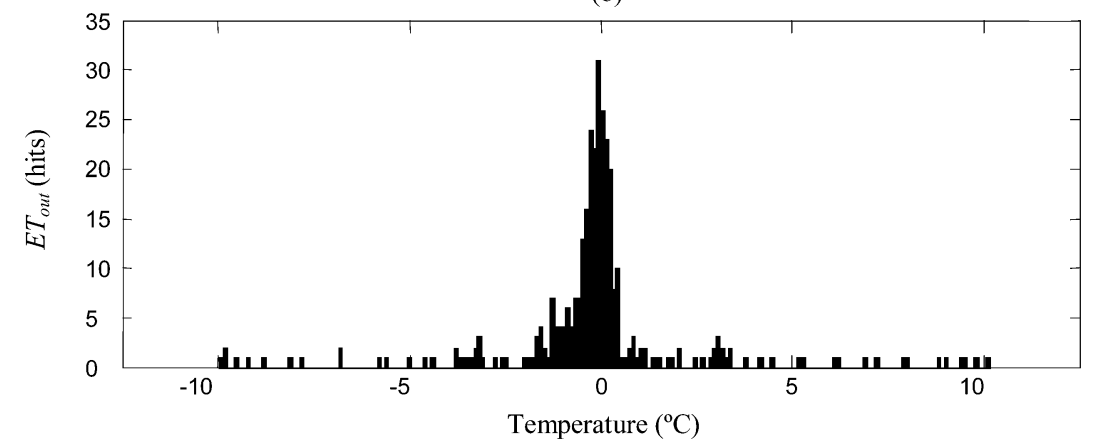

Fig. 11. Tout error histograms. (a) Classical MBPC. (b) Triangular-shaped fuzzy MBPC.
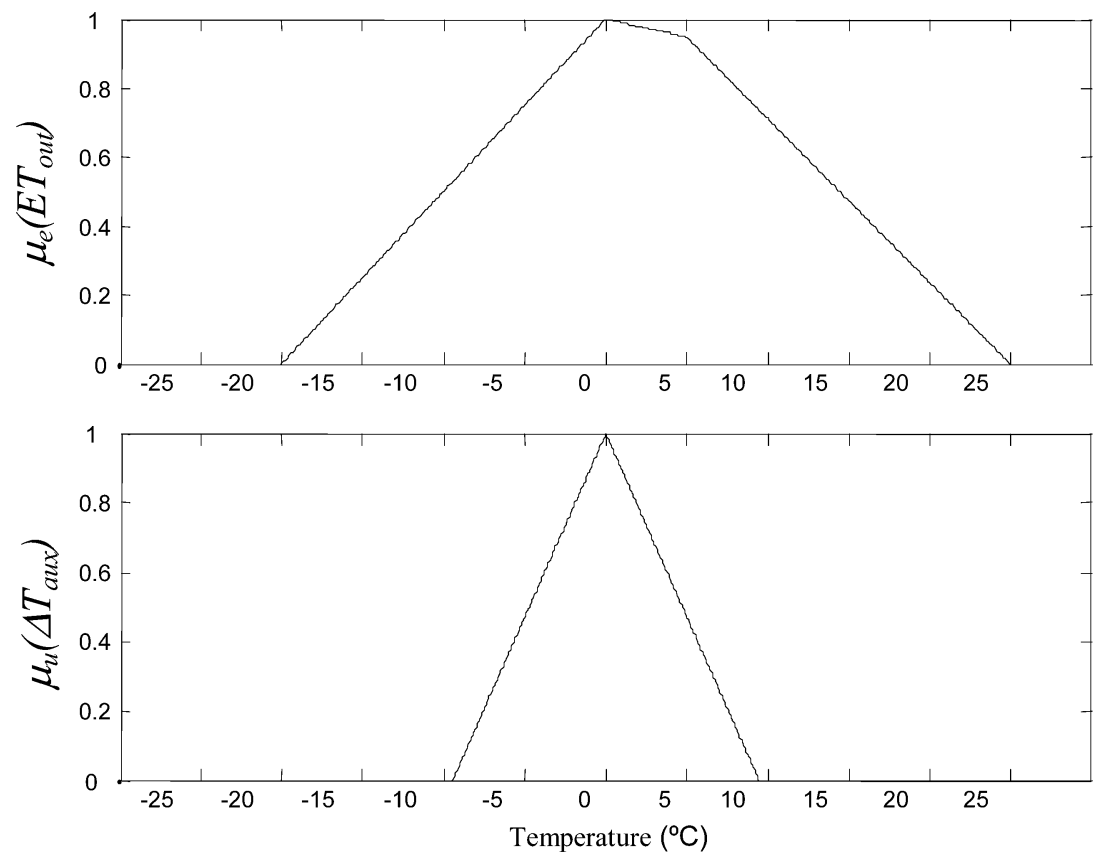

Fig. 12. Custom-shaped membership functions for $T_{\text {out }}$ error and $T_{\text {aux }}$ variation.

a second controller that uses custom-shaped membership functions is presented.

It will be assumed that one of the goals of the solar power plant is to maximize the heat transfer from the collector field to an unknown load. Under this assumption, the outlet oil temperature set-point is considered to be more strict when the controlled variable is under this reference and not so much in the opposite case (always considering that the maximum temperature will not surpass the physical limits of the installation, $300^{\circ} \mathrm{C}$ ). Unlike the classical MBPC, the fuzzy predictive controller can easily incorporate this kind of control goals.
The membership function for the error in the outlet oil temperature, $\mu_{e}$, is customized according to (14). The membership function for the oil flow variation is kept identical [see (12) and (13)]

$$
\operatorname{cumf}(x, a, b, c, d, p)= \begin{cases}\frac{x-a}{b-a}, & \text { if } a \leq x<b \\ 1+\frac{(1-p)(x-b)}{b-c}, & \text { if } b \leq x<c \\ \frac{p(x-d)}{c-d}, & \text { if } c \leq x \leq d \\ 0, & \text { otherwise. }\end{cases}
$$


(a)

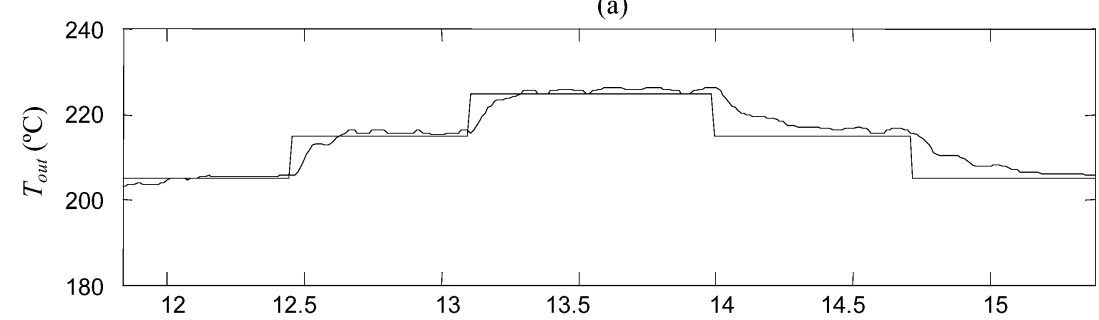

(b)

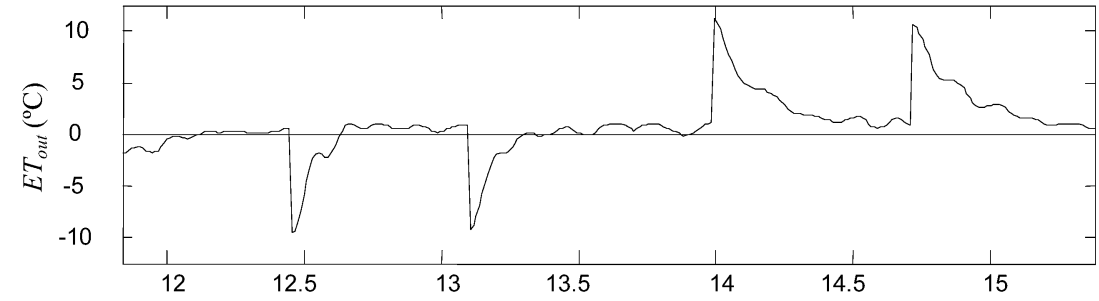

(c)

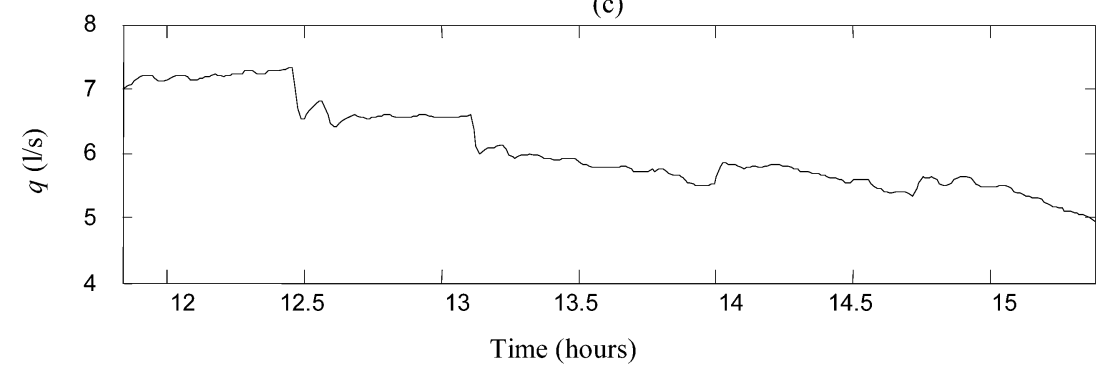

Fig. 13. Simulation results for fuzzy MBPC with custom-shaped membership functions. (a) Outlet oil temperature. (b) Error in the outlet oil temperature. (c) Oil flow.

(a)

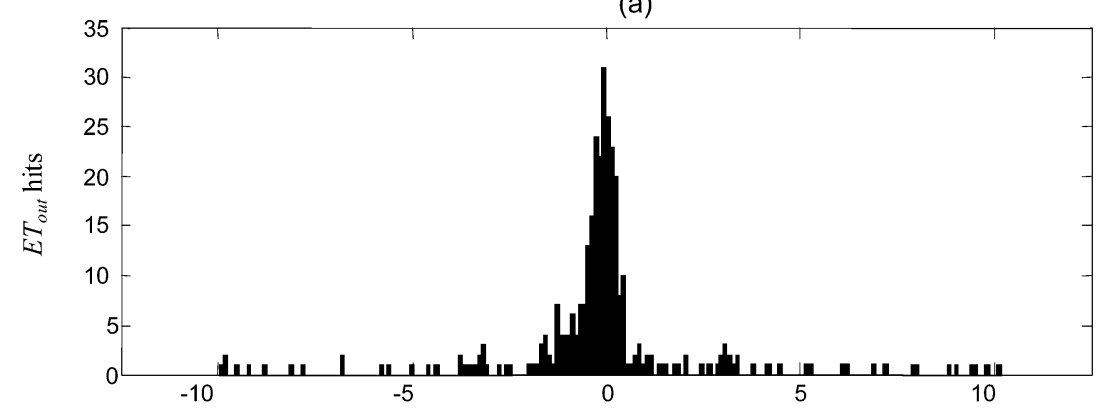

(b)

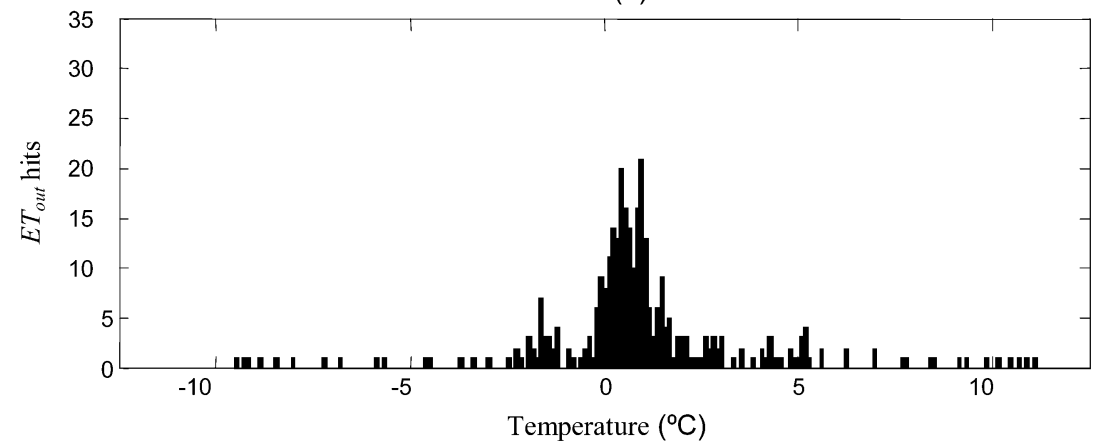

Fig. 14. $T_{\text {out }}$ error histograms. (a) Classical MBPC. (b) Custom-shaped Fuzzy MBPC.

New membership functions will be considered (see Fig. 12)

$$
\begin{aligned}
\mu_{e}\left(E T_{\text {out }}\right) & =\operatorname{cumf}\left(E T_{\text {out }},-20,0,5,25,0.99\right) \\
\mu_{u}\left(\Delta T_{\text {aux }}\right) & =\operatorname{trimf}\left(\Delta T_{\text {aux }},-9.5,0,9.5\right)
\end{aligned}
$$

The simulation results for this new fuzzy predictive controller with custom-shaped membership functions are shown in Fig. 13. The response of the plant with this controller is different to the previous two. Comparing these results to those in Figs. 8 and 
10 , it can be seen that the response times when the set-point is increased are smaller than those when the set-point is decreased. Additionally the manipulated variable, oil flow, is not as oscillatory as with the previous controllers.

After each set-point change there is a positive error that is not present in the previous controllers. This behavior is completely consequent with the fuzzy membership function for the outlet oil temperature error. The modifications on the error profile distribution can be appreciated looking at the error histograms in Fig. 14. According to Fig. 14(b), the reduction of negative error and the increase of positive error in the range $0^{\circ} \mathrm{C}$ to $5^{\circ} \mathrm{C}$ are concordant with the shape of the custom membership function $\mu_{e}$.

\section{CONCLUSION}

In order to apply classical control techniques such as MBPC to real processes, values of several parameters of the objective function and constraint limits must be chosen and sometimes this represents a difficult decision for the control engineer. As an alternative, it has been proposed the use of fuzzy membership functions to represent goals and constraints in a more flexible and transparent way.

A comparison has been made between classical MBPC and the new fuzzy methodology proposed applied to a solar power plant. The results show that under certain circumstances (weights, fuzzy membership functions, and fuzzy $t$-norm) the solutions of both control algorithms are quite similar.

However, the fuzzy MBPC formulation gives a greater flexibility to characterize the goals and constraints for the process. This fact can be appreciated in the performance of a fuzzy predictive controller based on custom-shaped membership functions.

As concluding remarks of the new methodology proposed we highlight two attributes: 1) It is based on a well-proven successful algorithm such a classical MBPC, and 2) It empowers MBPC with flexibility to consider goals and constraints different to those usually used in classical MBPC. This last remark is the main advantage of fuzzy predictive control. The flexibility provided by the fuzzy membership function and the fuzzy aggregation can be used to consider complex goals such as different weights for distinct error situation as shown in this paper. Other possible complex goals for the solar power plant could be simultaneous fulfillment of conditions in the outlet oil temperature and the oil flow.

Clearly, the possibilities of fuzzy predictive control are enormous and the role of the control engineer is to search for processes suitable for its application.

\section{REFERENCES}

[1] K. J. Aström and B. Wittenmark, Computer Controller Systems, Theory and Design. Upper Saddle River, NJ: Prentice-Hall, 1984.

[2] F. R. Rubio, F. Gordillo, and M. Berenguel, "LQG/LTR control of the distributed collector field of a solar power plant," in Proc. 13th Triennial World Congr., San Francisco, CA, pp. 133-138.

[3] E. F. Camacho, M. Berenguel, and F. R. Rubio, Advanced Control of Solar Plants. London, U.K.: Springer-Verlag, 1997.
[4] E. F. Camacho, F. R. Rubio, and F. Hughes, "Self-tuning control of a solar power plant with distributed collector field," IEEE Control Syst. Mag., vol. 12, no. 2, pp. 72-78, Feb. 1992.

[5] E. F. Camacho, M. Berenguel, and C. Bordons, "Adaptive model predictive control of a distributed collector field," IEEE Trans. Control Syst. Technol., vol. 2, no. 4, pp. 462-467, Apr. 1994.

[6] E. F. Camacho, M. Berenguel, and F. R. Rubio, "Application of a gain scheduling generalized predictive controller to a solar power plant," Control Eng. Pract., vol. 2, no. 2, pp. 227-238, 1994.

[7] E. F. Camacho and M. Berenguel, "Application of generalized predictive control to a solar power plant," in Proc. 3rd IEEE Conf. Control Applications, vol. 3, Glasgow, U.K., 1994, pp. 1657-1662.

[8] M. Berenguel, M. R. Arahal, and E. F. Camacho, "Modeling free response of a solar plant for predictive control," Control Eng. Pract., vol. 6, pp. 1257-1266, 1998.

[9] F. R. Rubio, M. Berenguel, and E. F. Camacho, "Fuzzy logic control of a solar power plant," IEEE Trans. Fuzzy Syst., vol. 3, no. 4, pp. 459-468, Aug. 1995.

[10] F. Gordillo, F. R. Rubio, E. F. Camacho, M. Berenguel, and J. Bonilla, "Genetic design of a fuzzy logic controller for a solar power plant," in Proc. 4th Eur. Control Conf., 1997, Paper NTH-E 15, pp. 283-288.

[11] A. Cardoso, J. Henriques, and A. Dourado, "Fuzzy supervisor and feedforward control of a solar power plant using accesible disturbances," in Proc. Eur. Control Conf., Karlsruhe, Germany, 1999, pp. 811-819.

[12] J. Henriques, A. Cardoso, and A. Dourado, "Fuzzy supervisor of PID controllers for the Acurex field at PSA," in Proc. 2nd Annu. Workshop Plataforma Solar de Almería, Almería, Spain, 1998, pp. 7-12.

[13] - "Supervision and $c$-means clustering of PID controllers for a solar power plant," Int. J. Approx. Reason., vol. 22, no. 1-2, pp. 73-91, 1999.

[14] E. Juuso, P. Balsa, and L. Valenzuela, "Multilevel linguistic equation controller applied to a $1 \mathrm{MW}$ solar power plant," in Proc. Amer. Control Conf., 1998, pp. 3891-3895.

[15] R. Pickardt, "Adaptive control of a solar power plant using a multimodel," Proc. Inst. Elect. Eng. Control Theory Applicat., vol. 147, no. 5, pp. 493-500, 2000.

[16] - "Application of adaptive controllers to a solar power plant using multi-model description," in Proc. Amer. Control Conf., 1998, pp. 2895-2899.

[17] T. Johansen, K. J. Hunt, and I. Petersen, "Gain scheduled control of a solar power plant," Control Eng. Pract., vol. 8, no. 9, pp. 1011-1022, 2000.

[18] E. F. Camacho, F. R. Rubio, and J. Gutierrez, "Modeling and simulation of a solar power plant with a distributed collectors system," in Proc. IFAC Symp. Power Systems, Modeling, and Control Aplications, Brussels, Belgium, 1988, pp. 11.3.1-11.3.5.

[19] E. F. Camacho and C. Bordons, Model Predictive Control. London, U.K.: Springer-Verlag, 1999.

[20] N. Oliveira and L. Biegler, "Constraint handling and stability properties of model-predictive control," AIChE J., vol. 40, no. 7, pp. 1138-1155, 1994.

[21] J. Sousa, R. Babuška, B. Bruijin, and H. Verbruggen, "Comparison of conventional and fuzzy predictive control," in Proc. 5th IEEE Int. Conf. Fuzzy Systems, New Orleans, LA, 1996, pp. 1782-1787.

[22] J. Sousa, U. Kaymak, and H. Verbruggen, "A comparative study of fuzzy and conventional criteria in model-based predictive control," in Proc. 6th IEEE Int. Conf. Fuzzy Systems, Barcelona, Spain, 1997, pp. 907-914.

[23] R. E. Bellman and L. A. Zadeh, "Decision making in a fuzzy environment," Manage. Sci., vol. 17, no. 4, pp. 141-164, 1970.

[24] U. Kaymak, J. Sousa, and M. Setnes, "Adaptive decision alternatives in fuzzy predictive control," in Proc. 7th IEEE Int. Conf. Fuzzy Systems, Anchorage, AK, 1998, pp. 698-703.

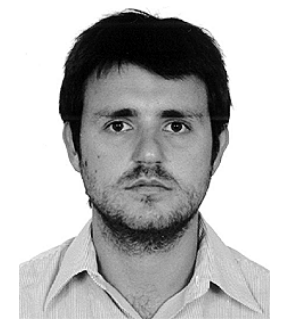

Andrés Flores received the M.Sc. degree in electrical engineering from the Pontificia Universidad Católica de Chile, Santiago, in 2001.

His area of research was focused on the application of fuzzy logic and artificial neural networks to model based predictive control. He is currently working as a Project and Support Engineer for Sony Professional Products, Santiago, Chile. 


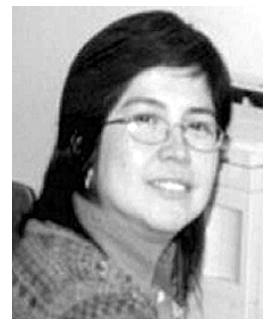

Doris Sáez (M'95) received the M.Sc. and Ph.D. degrees in electrical engineering from the Pontificia Universidad Católica de Chile, Santiago, in 1995 and 2000, respectively.

She is currently an Assistant Professor at the Departamento de Ingeniería Eléctrica, Universidad de Chile, Santiago. Her research interests include fuzzy systems control design, fuzzy identification, neural networks, and control of power generation plants.

Dr. Sáez is a Co-Founder of the Chilean chapter of the IEEE Neural Networks Society.

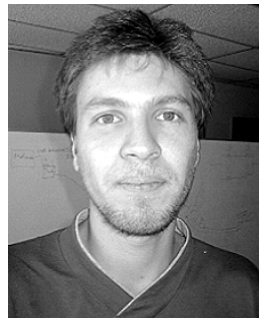

Juan Araya received the M.Sc. degree in electrical engineering from the Pontificia Universidad Católica de Chile, Santiago, in 2004.

His research interests include fuzzy systems, system identification, fault detection and diagnosis, and time-varying systems, including their application to industrial processes.

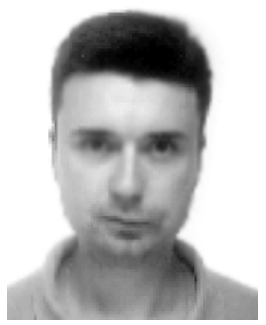

Manuel Berenguel received the Industrial Engineering and Ph.D. degrees from the Escuela Superior de Ingenieros Industriales, the University of Seville, Seville, Spain, in 1992 and 1996, respectively.

$\mathrm{He}$ is an Associate Professor in the Departamento de Lenguajes y Computación (Área de Ingeniería de Sistemas y Automática), the University of Almería, Almería, Spain, where he was a Researcher and Associate Professor in the Departamento de Ingeniería de Sistemas y Automática for six years. His research interests are in the fields of predictive, adaptive, and robust control, with applications to solar energy systems, agriculture, and biotechnology. He has authored and coauthored more than 50 technical papers in international journals and conferences, and is coauthor of the book Advanced Control of Solar Plants (New York: Springer-Verlag, 1997).

Dr. Berenguel he received the "Premio Extraordinario de Doctorado" Award (given to the best engineering thesis of the year). He has acted as a Reviewer for such journals as Control Engineering Practice, the IEEE TRANSACTIONS ON SySTEMS, MAN, AND CYBERNETICS, and the IEEE TRANSACTIONS ON FUZZY SYSTEMS.

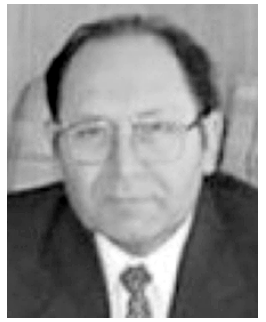

Aldo Cipriano (M'74-SM'95) received the electrical engineering and M.Sc. degrees, both from the Universidad de Chile, Santiago, in 1973 and 1974, respectively, and the Dr.Ing. degree from the Technical University of Munich, Munich, Germany, in 1981.

Since 1974, he has been with the Pontificia Universidad Católica de Chile, Santiago, where he is currently a Professor of Electrical Engineering. From 1998 to 2003, he was the Dean of the College of Engineering at the same university. In 1990, he was a Visiting Professor at University of Karlsruhe, Karlsruhe, Germany. He has been involved in the design and implementation of a variety of advanced control systems for mineral processing plants. His current research interests include fault detection and diagnosis, predictive control, and fuzzy systems.

Dr. Cipriano served as chairman of the Chilean Association of Automatic Control (1983-1985) and of the Chilean Joint Chapter of the IEEE Control Systems-Industrial Electronics Societies (1983). He was Co-Chairman of the 1994 IEEE International Symposium on Industrial Electronics and chairman of the IFAC Technical Committee on Low Cost Automation (1996-1998). 